\title{
Modellierung der Ausbreitung von Baumschädlingen nach aerochemischer Insektizidanwendung mit den Mitteln eines Geoinformationssystems
}

\author{
Colja Krugmannn', Majdi Abusaleh¹, René Krüger ${ }^{1}$, J ochen Wittmann ${ }^{1}$ \\ ${ }^{1}$ Hochschule für Technik und Wirtschaft Berlin, Umweltinformatik, \\ Wilhelminenhofstraße 75A, 12459 Berlin, Germany, wittmann@ htw-berlin.de
}

\begin{abstract}
Im Rahmen eines Studentenprojektes für das Modul Umwelt- und Geoinformationssysteme greift der vorliegende Beitrag die Diskussion um eine aerochemische Bekämpfung von Waldschädlingen auf und versucht allein auf der Basis von öffentlich zugänglichen Daten ein Modell zu entwickeln, das die für eine Besprühung erlaubten Flächen aufzeigt, das aber darüber hinaus auch versucht, im Rahmen der Entwicklung eines dynamischen Modells die emeute Ausbreitung der Waldschädlinge nach der Bekämpfungsmaßnahme einzuschätzen. Grundlegende Annahme für diese Modellierung ist die Tatsache, dass bei einer Besprühung Schutzzonen um besiedeltes $\mathrm{Gebiet}$ aber auch um Oberflächengewässer und Waldränder eingehalten werden müssen, die nicht behandelt werden dürfen und damit zu Zonen werden, in denen die Schädlinge überleben. Nimmt man nun noch eine bestimmte Dauer für den Vermehrungszyklus der Schädlinge sowie einen mittleren Verbreitungsradius an, kann man mit einfachen Methoden eines Geoinformationssystems die dynamische Ausbreitung der Schädlingspopulation über mehrere Vermehrungszyklen simulieren und darstellen. Das Paper zeigt als Machbarkeitsstudie, dass ein solches, einfaches Modell sinnvolle Ergebnisse liefern kann und stellt für konkrete Anwendungen eine Liste mit den unbedingt notwendigen Parametern auf, die erhoben werden müssen, um eine praxisnahe Simulation durchführen zu können.
\end{abstract}

\section{Motivation}

2019 wurde in $\mathrm{B}$ randenburg seit langem wieder in $\mathrm{Er}$ wägung gezogen, aerochemisch mit "K arate Forst flüssig" von Syngenta gegen W aldschädlinge, in erster Linie gegen die N onne, vorzugehen. V on Seiten von U mweltschützer*innen und A nwohner*innen gab es regen W iderstand ( $M$ it "Karate Forst" gegen Raupen in $B$ randenburgs Wäldern, 2019). B randenburgs Wälder bestehen zu $70 \%$ aus $\mathrm{K}$ iefern. Diese M onokultur zusammen mit den dürren Sommern der vergangenen J ahre erhöhte die A nfälligkeit der B äume für Schadorganismen wie K iefernspinner, Kiefernspanner, K iefernbuschhornblattwespe, Forleule und Nonne. Das Waldmonitoring prognostizierte bei günstigen Bedingungen für die Schädlinge wie warmes und trockenes W etter eine große G efahr des $K$ ahlfraßes. K iefern können einen einmaligen $K$ ahlfraß von ca. $90 \%$ mit wenig V erlusten überstehen, kommt es jedoch zu stärkeren Schäden ist von einem $A b$ sterben des $W$ aldes auszugehen (Landesbetrieb Forst B randenburg (Hrsg.), kein Datum). A uch in B ezug auf den $\mathrm{CO} 2-\mathrm{H}$ aushalt stellen die W älder einen wesentlichen Faktor dar und ein $\mathrm{K}$ ahlfraß würde die Forste von einer positiv zu bewertenden $\mathrm{CO}$ 2-Senke in eine für die B ilanz negative CO2-Quelle verwandeln. Dem allen wollte das Landeskompetenzzentrum Forst Eberswalde (LFE) mit aerochemischer Schädlingsbekämpfung mit K arate Forst flüssig von Syngenta begegnen (Landesbetrieb Forst B randenburg ( $\mathrm{H} \mathrm{rsg.)}$, kein Datum). B ei diesem Insektizid handelt es sich um ein K ontaktgift, das hoch verdünnt in die Baumkronen der befallenen Gebiete ausgebracht wird. Es tötet die Nonne im Raupenstadium (L andesbetrieb Forst B randenburg (Hrsg.), kein Datum).

$N$ aturschützer zweifelten stark an der Sinnhaftigkeit und U mweltverträglichkeit dieser $\mathrm{V}$ orgehensweise, da auch die natürlichen Feinde der Schädlinge mit betroffen 
wären. Laut LFE kann die K onzentration aber gerade so hoch eingestellt werden, dass allein die Nonnenraupen abgetötet werden und nicht deren Gegenspieler oder beispielsweise M aikäfer oder andere L aufkäfer.

Die vorliegende M achbarkeitsstudie greift an dieser Stelle in die Diskussion ein. Es soll geprüft werden, ob man durch eine Modellentwicklung, die ausschließlich auf öffentlich zugänglichem Datenmaterial beruht, A ussagen über die A uswirkungen einer aerochemischen Besprühung auf die Entwicklung des Schädlingsbefalls machen kann. B esondere B erücksichtigung bei der M odellierung soll die Bedeutung der Schutzzonen finden, die bei der A usbringung des Insektizids um Siedlungen, Gewässer usw. eingehalten werden müssen und damit Rückzugsgebiete für die Schädlingspopulation darstellen aus denen heraus sich diese sowohl zahlenmäßig erholen als auch neu verbreiten kann.

\section{Modellidee und Aufbereitung für eine GIS-Analyse}

Die Grundidee der vorliegenden Arbeit ist es zunächst, ausschließlich öffentlich verfügbares $K$ artenmaterial als $B$ asisdaten für den A nwendungsfall aufzubereiten, das die für eine B esprühung erlaubten und geeigneten Flächen aufzeigt, das aber dar-über hinaus auch versucht, im Rahmen der Entwicklung eines dynamischen M odells die Verbreitung der Waldschädlinge nach der Bekämpfungsmaßnahme einzuschätzen. Grundlegende A nnahme für diese M odellierung ist die Tatsache, dass bei einer B esprühung Schutzzonen um besiedeltes $G$ ebiet aber auch um 0 berflächengewässer und $W$ aldränder eingehal ten werden müssen, die nicht behandelt werden dürfen und damit als Rückzugsgebiete für die Schädlingspopulation dienen können. Nimmt man nun noch eine bestimmte Dauer für den Vermehrungszyklus der Schädlinge sowie einen mittleren Verbreitungsradius an, soll allein mit den Standardmethoden zur räumlichen A nalyse, die ein Geoinformationssystem zur Verfügung stellt, die dynamische A usbreitung der Schädlingspopulation über mehrere Vermehrungszyklen simuliert und dargestellt werden. (s. A bb. 1)

Ziel der Modellierung ist es dabei, den Einfluss und die B edeutung der Schutzzonen herauszuarbeiten und einer quantitativen Untersuchung zu öffnen. Denn sicherlich wird der Insektizideinsatz in einer topographisch stark strukturierten und damit stark mit Schutzzonen $\downarrow$

Ist-Situation in einer Karte darstellen

Ausbringen des Insektizides auf die erlaubten Flächen mit

$\downarrow$ vollständiger Vernichtung der Schad-Population

$\Rightarrow$ in

Simulation der Ausbreitung der verbleibenden Schad-Population in einem Vermehrungszyklus

Abb. 1: Modellidee

durchsetzten Landschaft andere Effekte zeigen als in einer großflächig strukturierten Landschaft, die weniger Schutzzonen ausweist.

M it dieser M odellidee als Basis stellen sich für die A nalyse im Geoinformationssystem die folgenden Teilaufgaben:

1. Finden und A ufbereiten geeigneter öffentlich zugänglicher G eobasisdaten

2. Ermittlung der für die M odellierung notwendigen Parameterwerte

a. B ezüglich der A nwendungshinweise des Insektizids

i. Schutzzone um Siedlungen

ii. Schutzzone um Gewässer

iii. Schutzzone an W aldrändern

b. Bezüglich der biologischen Daten des Schädlings

i. Dauer eines V ermehrungszyklus

ii. A usbreitungsreichweite in einem $V$ ermehrungszyklus

\section{Der Analyse-Workflow}

$\mathrm{N}$ ach der $\mathrm{V}$ eranschaulichung der $\mathrm{M}$ ethode folgt nun die Schilderung der einzelnen A rbeitsschritte. A Is Erstes wurde ein W egenetz benötigt. Als Zweites musste der Grenzwert bzw. M aximalwert an Emissionen berechnet werden, der den E rreichbarkeitsgrafen für die Transportmittel als L i imit dienen soll. I m D ritten und letzten Schritt konnten die E rreichbarkeitsgrafen dann erstellt werden.

\subsection{Schritt 0: Grundkarte und Geobasis da- ten erstellen}

Die für die $M$ achbarkeitsstudie getroffene B eschränkung auf öffentlich zugängliches $K$ artenmaterial stellt eine gewisse Herausforderung dar, die einerseits zwar zusätzliche Arbeitsschritte erfordert, andererseits jedoch 
die U nabhängigkeit des M odellansatzes von bereits vorliegendem, jedoch nur beschränkt und/oder unter A uflagen verfügbarem Datenmaterial unterstreicht.

Als B eispielfläche wurde eine Region in Brandenburg zwischen Bad B elzig und W erder (Havel) gewählt, für den sich die $D$ atenlage folgendermaßen darstellt:

- Als Grundkarte wird ein Ausschnitt aus openstreetmap gewählt (O penStreetM ap, 2019)

- Die G ewässerdaten als Shape-D ateien stammen vom Geoportal B randenburg (LGB (L andesver messung und Geobasisdaten Brandenburg), 2019).

- Die Straßennetzdaten für B randenburg stammen von Inspire Brandenburg und wurden über ein API in Form einer GM L-Datei heruntergeladen (LGB (Landesvermessung und Geobasisdaten B randenburg), 2019) .

- Die Walddaten stammen vom Landeskompe tenzzentrum Forst B randenburg und lagen als GML-Datei vor (L andesbetrieb Forst Branden burg (Hrsg.), kein Datum).

- Idealerweise liegen Siedlungen und landwirtschaftlich genutzte Flächen als Shape-Dateien, in vorliegendem Beispiel allerdings nur als Lü cken in den Forstgebieten vor.

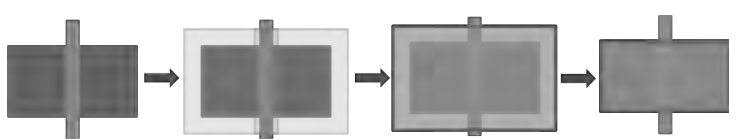

Abb. 2: Skizze zu Schritt 1: zusammenhängende Waldflächen erzeugen

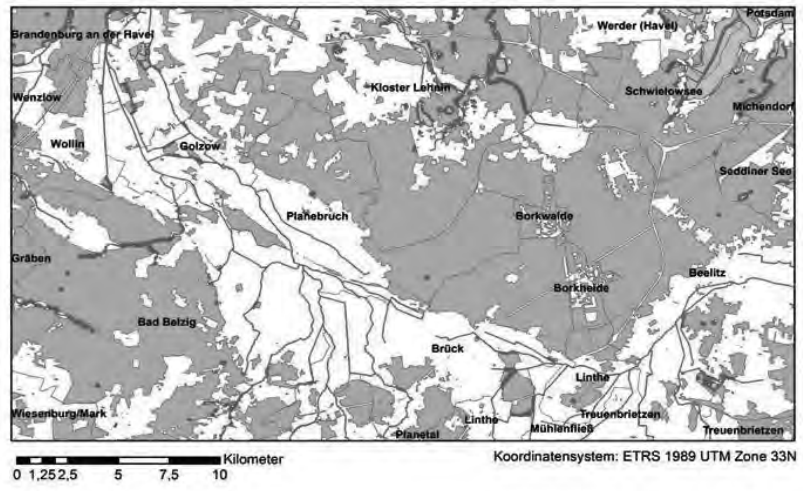

Abb. 3: Basiskarte nach Schritt 1

\subsection{Schritt 1: zusammenhängende Waldflächen erzeugen}

Innerhal $b$ eines Forstes befinden sich $W$ aldwege und kleinere Straßen, welche zu einer Unterbrechung der Forstfläche in den Geodaten führen und damit fiktive W aldränder darstellen, die als Schutzzonen relevant wären. In Bezug auf einen Insektizideinsatz zählen Waldwege jedoch nicht als Grenze eines Forstgebietes und müssen deswegen für dieses Projekt ignoriert werden, um mit geschlossenen Forstflächen weiterarbeiten zu können.

U m das zu erreichen, wird um die Forstgebiete ein Puffer gelegt, der die Waldwege überdeckt. Die daraus entstandenen überlappenden $W$ aldpolygone werden anschließend zusammengeführt (Dissolve). Ein anschließender negativer Puffer um die gleiche B reite reduziert die W aldgebiete schließlich auf ihre ursprüngliche, reale Größe. (Skizze des Verfahren Abb. 2, Basiskarte nach diesem B earbeitungsschritt in A bb. 3)

\subsection{Schritt 2: einheitliche Gewässerflächen mit Sicherheits abstand erzeugen}

Geodaten für Seen liegen in F orm von Polygonen und für Flüsse und $K$ anäle in Form von Linien vor. Linien haben keine Breite, weshalb sie vor einer Vereinigung mit den Seeflächen zunächst in Polygone umgewandelt werden müssen. Hierfür wurde ein Puffer um die Flüsse als line-Feature erstellt. A nschließend können Seen und Flüsse zu einem Layer "Gewässer" zusammengeführt werden, der für die Bestimmung der Schutzzonen relevant ist. Sämtliche $G$ ewässer-F eatures werden nun erneut um die B reite der Schutzzone gepuffert, damit Polygone entstehen, die die Gewässer inklusive ihres Sicherheitsabstands repräsentieren. (A bb. 4)

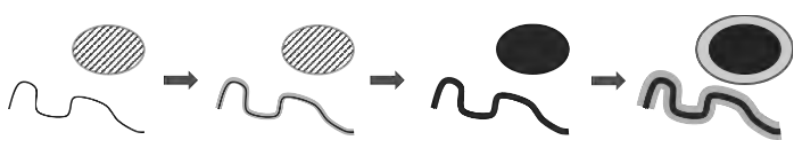

Abb. 4: Schritt 2: einheitliche Gewässerflächen mit Sicherheitsabstand erzeugen 


\section{4}

Schritt 3: Sicherheitsabstand zu großen Straßen; Zusammenführung der Schutzzonen

Ü ber größere Straßen, wie B undes- und K reisstraßen oder Bundesautobahnen darf nicht hinweg gesprüht werden. Die G eodaten für Straßen liegen wiederum als lineFeatures vor. U m Schutzzonen entlang von Straßen zu ermitteln werden diese wiederum entsprechend einer gemittelten Straßenbreite und des vorgeschriebenen Schutzabstands gepuffert. Diese Schutzzonen um Straßen werden anschließend mit den Schutzzonen um Gewässer zusammengeführt. (A bb. 5)

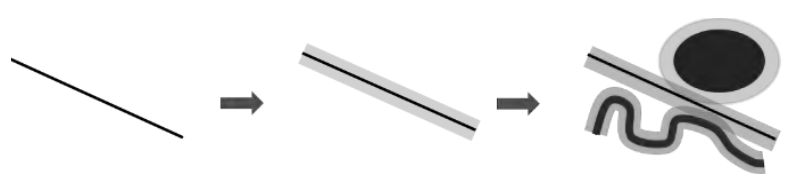

Abb. 5: Schritt 3: Sicherheitsabstand zu Straßen; Zusammenführung der Schutzzonen

\subsection{Schritt 4: nicht besprühte Waldfläche}

U m die Gebiete zu bestimmen, die nicht besprüht werden dürfen, wurde zunächst die besprühbare $W$ al dfläche identifiziert. Die W aldpolygone aus Schritt 1 werden um den Sicherheitsabstand zum $W$ aldrand negativ gepuffert. Diese Fläche wird mit den Flächen der Gewässer und großen Straßen aus Schritt 2 und 3 verschnitten. W ird das E rgebnis dieser $V$ erschneidung nun noch durch eine symmetrische Differenz von der gepufferten Waldfläche abgezogen, erhält man eine Waldfläche, bei der alle Sicherheitsabstände berücksichtigt wurden, al so die W al dfläche, die mit dem Insektizid besprüht werden darf. J etzt kann man erneut eine symmetrische Differenz mit der gesamten $W$ aldfläche bilden und man erhält schließlich diejenige $W$ aldfläche, die nicht besprüht werden darf. (A bb. 6)

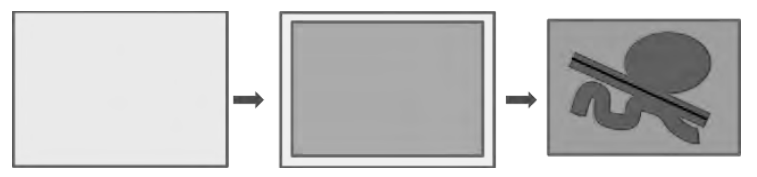

Abb. 6: Schritt 4: nicht besprühte Waldfläche ermitteln

\subsection{Schritt 5: Ausbreitung des Schädlings}

$M$ an geht davon aus, dass die Schädlinge in dem Gebiet, in dem nicht gesprüht werden darf, vollständig überleben. $V$ on diesem Gebiet aus können sie sich folglich erneut in die benachbarten, behandelten $\mathrm{G}$ ebiete ausbreiten. Dieser A usbreitungsprozess kann durch einen Puffer um die nicht behandelten G ebiete modelliert werden. Die Pufferbreite entspricht dabei der A usbreitungsgeschwindigkeit des Schädlings in einem V ermehrungszyklus. Da die angenommene Ausbreitung in alle Richtungen geht reicht sie über die $W$ aldgrenzen hinaus, weshalb sie miteinander verschnitten werden müssen. Zudem kann es zu einer Überlagerung von Teilen der Ausbreitungszone kommen, die durch ein Zusammenführen entfernt werden. (A bb. 7)

Nun kann diese gesamte Prozedur für genau einen V ermehrungszyklus wiederholt abgearbeitet werden, um die A bfolge und die A uswirkungen mehrerer V ermehrungszyklen zu simulieren.

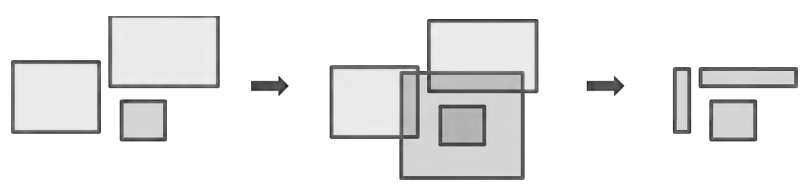

Abb. 7: Ausbreitung des Schädlings

\section{Ergebnisse}

\subsection{Machbarkeitsstudie für die Beis pielre- gion}

Die im vorausgegangenen $A$ bschnitt beschriebenen A nalyseschritte wurden mit dem Model Builder in Esris A rcM ap 10.5 als A nalyseworkflow angelegt und auf die $B$ eispiel region aus $A$ bbildung 3 angewandt. Für die diversen in den Einzelschritten genannten Parameter wurden die W erte plausibel geschätzt, die exakten Parameterwerte sollen an dieser Stelle jedoch bewusst nicht genannt werden, da es sich bei dem Modell ausdrücklich um einen Prototypen handelt, der auf den prinzipiellen A nalyseworkflow abzielt und die Ergebnisse daher zunächst nur einem Plausibilitätstest unterzogen werden können. Im nachfolgenden U nterabschnitt soll die Frage der Parametrisierung separat diskutiert werden.

Als Ergebnis der Modellierung und der Simulation 
können daher zunächst nur drei qualitative Schlüsse gezogen werden: Erstens ist der beschriebene schrittweise A nalyseworkflow mit den Standardmethoden von A rc$M$ ap vollständig und transparent abbildbar und steht als frei parametrisierbares neues Tool zur V erfügung. Zweitens ist es möglich, eine einfache dynamische Simulation durch I teration der in Schritt 5 erläuterten A usbreitungsmethode zu implementieren. Und drittens führt das durch den Workflow beschriebene Vorgehen tatsächlich zu plausiblen Ergebnissen, wie aus der A bbildung 8 ersichtlich ist.

$M$ an erkennt in der Ergebniskarte, dass bereits nach drei defensiv parametrisierten Vermehrungszyklen (gekennzeichnet durch unterschiedlich stark rot symbolisierte Pufferflächen um die Schutzzonen) ein großer Teil der W aldfläche ausgehend von den Schutzzonen neu besiedelt ist. Die A bhängigkeit von der $L$ andschaftsstruktur wird durch das Modell offensichtlich nachvollziehbar.

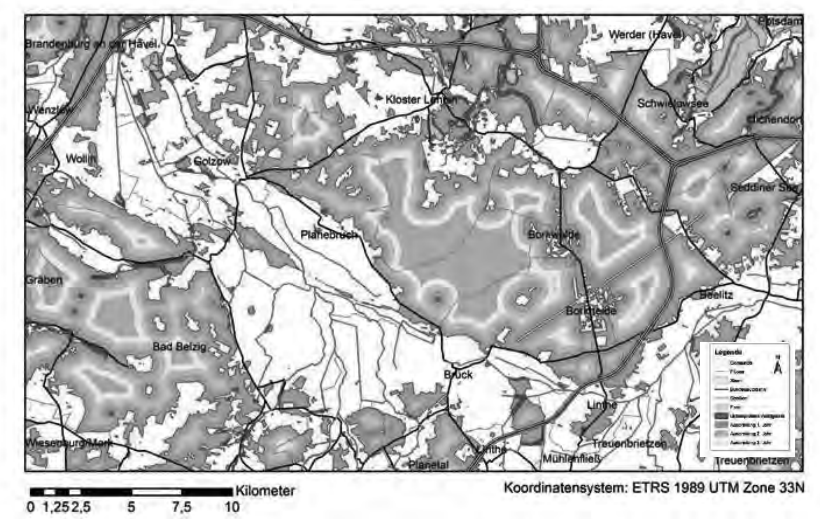

Abb. 8: Simulierte Situation für die Beispiel region nach drei Ausbreitungszyklen

\subsection{Notwendige Parameterwerte für eine exakte Modellstudie}

W ie bereits erwähnt, basiert das vorgestellte M odell auf öffentlich zugänglichen Daten, ist aber an einigen Stellen von Parameterwerten abhängig, die in der $\mathrm{M}$ achbarkeitsstudie lediglich plausibel geschätzt werden konnten für eine auch quantitativ verwertbare M odelluntersuchung aber mit validen W erten besetzt werden müssen. A llerdings ist anzumerken, dass die M odellierung darauf abzielte, dass es sich um eine verhältnismäßig kleine Zahl von Parametern handelt, die zudem relativ einfach zu bestimmen zu sein scheinen. Hier die L iste dieser M o- dellparameter, die für eine konkrete A nwendung zahlenmäßig zu besetzen sind:

1.Geobasisdaten:

a. Grundkarte (die M achbarkeitsstudie arbeitet bewusst ausschließlich mit öffentlich zugänglichem $M$ aterial, hier sind sicherlich bessere thematische B asiskarten möglich)

b. B reite von kleinen Fließgewässern

c. B reite von Straßen

2. A nwendungshinweise des Insektizids:

a. M indestabstand zu Waldrand

b.M indestabstand zu O berflächengewässern

c. M indestabstand zu Siedlungen

d. Wirksamkeit

3. Daten zur B iologie des Schädlings:

a. Dauer eines Vermehrungszyklus

b. A usbreitungsreichweite in einem Vermehrungszyklus

Dies ist die vollständige Parameterliste, die für das vorgestellte Modell benötigt wird. Selbstverständlich sind für eine detailliertere Modellierung noch viele andere Einflussfaktoren denkbar und sinnvoll. B ei der B eschäftigung mit dem M odell stößt man zum B eispiel sehr schnell auf den Einfluss des W etters und die Zusammensetzung des B aumbestandes als zwei Größen, die eigentlich nicht unberücksichtigt bleiben dürften, die aber mit Blick auf das Ziel, zunächst nur die prinzipielle $\mathrm{M}$ achbarkeit zu demonstrieren, trotzdem vernachlässigt wurden.

\section{Fazit}

Die vorliegende A rbeit soll zeigen, dass bereits ein sehr einfacher M odellansatz aussagekräftige Ergebnisse generieren kann, die es erlauben, die A uswirkungen eines Eingriffs durch aerochemische Insektizidanwendung abzuschätzen und die Fläche der unbehandelten Waldgebiete zu bestimmen. $M$ it einer überschaubaren $M$ enge von dazu benötigten Parameterwerten und einem sowohl algorithmisch als auch softwaretechnisch geringen Aufwand lässt sich durch Parametervariationen und Szenariountersuchungen die Diskussion sicherlich versachlichen.

A uf methodologischer Ebene zeigt das vorgestellte Modell, dass die häufig diskutierte Kopplung und/oder 
Integration von Geoinformationssystem und Simulationssystem bei einem engen Anforderungsprofil einerseits und einem sehr pragmatischen M odellierungsansatz andererseits für Einzelprojekte pragmatisch und erfolgreich gelingen kann.

\section{Quellen}

Geofabrik. (09.. 07. 2019). O penStreetM ap Europe. V on https://download.geofabrik.de/europe.html. abgerufen

L andesbetrieb Forst B randenburg (H rsg.). (kein Datum). Abgerufen am 03. 042020 von $W$ aldschutzmaßnahmen gegen N onnenraupen: https://forst.brandenburg.de//fb/de/lfe/waldsch utzinformationen/wal dschutzmassnahmengegen-nonnenraupen/

Landesbetrieb Forst Brandenburg. (17. 01 2018). F orstgrunddaten - Flaechen - Waldbedeckung Land Brandenburg. A bgerufen am 23. 042019 von $\quad h t t p: / / w w w . b r a n d e n b u r g-$ forst.de/inspire/dls/ifgk_wld/

LGB (L andesvermessung und Geobasisdaten B randenburg). (2019). G eoportal Brandenburg. Abgerufen am 14. $05 \quad 2019$ von https://geoportal.brandenburg.de/startseite/

M ajunke, C., M öller, K., \& Funke, M . (2004). Die N onne (Lymantria monacha L., Lepidoptera, Lymantriidae). (L. Eberswalde, Hrsg.) Walschutz-M erkblatt 52.

$M$ it "Karate Forst" gegen Raupen in Brandenburgs Wäldern. (26. 04 2019). A bgerufen am 03. 04 2020 von rbb24.de: https://www.rbb24.de/panorama/beitrag/2019/0 4/brandenburg-beelitz-mit-karate-forstfluessig-gegen-insekten.html

O penStreetM ap. (2019).

https://www.openstreetmap.org abgerufen 